\title{
Caractérisation de la population Trotteur Français d'après leur estimation génétique par un BLUP modèle animal
}

\section{A. Tavernier}

INRA, Station de génétique quantitative et appliquée, 78350 Jouy-en-Josas, France

(reçu le10 février 1989, accepté le 30 juin 1989)

\begin{abstract}
Résumé - La valeur génétique des chevaux de race Trotteur Français est maintenant estimée par un BLUP. Cette méthode permet de prendre en compte toutes les performances de tous les apparentés du cheval et de tenir compte des différentes conditions de milieu : effet maternel, élevage, âge, sexe, année de la performance, conditions d'exploitation. Cent quarante mille cent sept Trotteurs Français ont été estimés. La performance utilisée est le logarithme du gain annuel divisé par le nombre de départs. L'indice est exprimé en points d'écart type phénotypique $\left(\sigma_{0}=20\right)$. De cet indice, se dégage la structure de la population. Les meilleurs élevages se trouvent en BasseNormandie et dans le Maine. La distribution des indices, en 1986, a pour moyenne +11 et pour écart type 6. La progression génétique du Trotteur est de 3,5\% d'écart type phénotypique par an. La différence de valeur entre la jumenterie qui est exploitée en course et celle qui ne l'est pas est très faible. L'homogamie entre indices est de 0,44. Le BLUP doit devenir un outil de sélection plus privilégié de la population; il permet, avec un intervalle de génération raccourci, une augmentation du progrès génétique de $50 \%$.
\end{abstract}

chevaux Trotteurs - gain - BLUP — Indice - sélection

Summary - Breeding characteristics of the French Trotter using a BLUP animal model. The breeding value of the French Trotter is now estimated using the BLUP method. This method takes into account all records of all relatives of a horse and environmental effects such as maternal effect, herd effect, age, sex, year of recording and permanent environmental effect. One hundred and forty thousand one hundred and seven French Trotters are evaluated. Records are the logarithm of annual earnings divided by the number of starts. Breeding evaluation is expressed setting a value of 20 to one phenotypic standard deviation. Characteristics of the population are stressed. The best herds are in Lower Normandy and in Maine. Births of 1986 have a mean of +11 and a standard deviation of 6 . Genetic trend is $3.5 \%$ of the phenotypic standard deviation per year. Broodmares without records are not worse than those recorded. The correlation between mates is 0.44 , evidencing a large non-random mating. BLUP evaluation should become the selection tool and will give, with shorter generation interval, a genetic trend with over $50 \%$ improvement.

trotters - earnings - BLUP — breeding evaluation - selection 


\section{INTRODUCTION}

L'estimation de la valeur génétique des chevaux de course de trot suscite, en Europe et aux Etats-Unis, un intérêt généralisé. Que ce soit sur le record (meilleure réduction kilométrique) ou les gains, des programmes d'indexation sont mis en place (Arnason et al., 1988; Katona \& Distl, 1988; Klemetsdal, 1988; Langlois, 1986, 1988; Leroy, 1988; Minkema, 1988; Ojala, 1988; Tavernier, 1988c; Tolley et al., 1988).

Les chevaux de race Trotteur Français sont indexés, depuis quelques années déjà, sur leurs gains en course grâce à un indice classique qui combine les performances propres pour les chevaux titulaires de performances ainsi que celles des descendants et des conjointes pour les étalons. Pour parfaire l'estimation génétique des Trotteurs, nous utilisons maintenant le BLUP (Best Linear Unbiased Predictor ) en modèle animal. Cette méthode d'évaluation nous permet d'intégrer toutes les performances de toute la parenté d'un cheval pour estimer son potentiel comme reproducteur. Cette approche est plus fidèle à la pratique des éleveurs. De plus, elle intègre simultanément les effets du milieu et les effets génétiques, ce qui améliore leur estimation, notamment en cas de distribution différentielle des niveaux génétiques dans les effets du milieu. L'indice est plus précis, plus juste et d'une gestion plus simple avec un mode de calcul uniforme, comme pour les chevaux de concours hippique. C'est donc un outil particulièrement efficace pour gérer la sélection d'une population : sélection des étalons, des poulinières et choix des accouplements. C'est aussi une source d'informations sur la structure actuelle de la population : localisation des meilleurs élevages, possibilité d'amélioration de la race, évaluation des juments non titulaires de performances. Ces caractéristiques permettent de préciser le niveau actuel de la population Trotteur Français et ses possibilités d'avenir.

\section{MATÉRIEL ET MÉTHODES}

La technique de calcul utilisée a été décrite pour les chevaux de sport (Tavernier, 1988b). Nous n'en ferons donc qu'un bref rappel. Le choix du critère de mesure de la performance et des facteurs de variation a été discuté par Langlois $(1984,1986)$.

\section{Modèle}

La performance en course est mesurée par le logarithme du gain annuel du cheval divisé par son nombre de départs (Langlois, 1984). Le logarithme permet de rétablir l'échelle distendue des gains, puisque ceux-ci sont distribués de façon exponentielle par rapport au niveau de la performance : un cheval gagne environ $45 \%$ de ce que gagne le cheval placé avant lui. Le gain est la meilleure mesure de la réussite du cheval en course. Cette performance est expliquée par le modèle linéaire :

$$
y=X b+Z u+W m+Z p+e
$$

avec :

- y : vecteur des observations (logarithme du gain annuel par départ);

- $\mathbf{b}$ : vecteur des effets fixes (sexe et classe d'âge-année);

$-u$ : vecteur des valeurs génétiques additives individuelles;

$-m$ : vecteur des effets "maternels-élevage»;

- $p$ : vecteur des effets du milieu commun aux différentes performances d'un même cheval;

$-e$ : vecteur de l'erreur résiduelle;

$-X, Z, W$ : matrices d'incidence.

Les effets connnus du milieu (effets fixes) sont le sexe et une combinaison de l'âge et de 
l'année. II y a 5 classes d'âge : $2,3,4,5$ ans et 6 à 10 ans, La combinaison de l'âge et de l'année est nécessaire, car la politique de dotation des courses en fonction de l'âge varie dans le temps et se combine aux effets de l'inflation. L'effet "maternel-élevage" est une interprétation de la différence trouvée entre les composantes paternelle et maternelle de la variance. Cette différence est due à un effet de milieu commun aux différents descendants d'une même mère. Soixante pour cent des élevages ne comprennent qu'une seule jument; l'effet maternel strict se confond donc avec l'influence de l'éleveur : alimentation, soins, mise en valeur des produits. L'effet de milieu commun aux différentes performances d'un même cheval représente la différence entre la corrélation entre 2 performances (répétabilité) et l'héritabilité. II est expliqué par le milieu constant dont bénéficie le cheval plusieurs années de suite.

Les espérances des effets de ce modèle linéaire sont :

$E\left[\begin{array}{c}y \\ u \\ m \\ p \\ e\end{array}\right]=\left[\begin{array}{c}X b \\ 0 \\ 0 \\ 0 \\ 0\end{array}\right]$

La matrice de variance-covariance est :

$V\left[\begin{array}{l}u \\ m \\ p \\ e\end{array}\right]=\left[\begin{array}{cccc}A \sigma_{\mathrm{u}}{ }^{2} & 0 & 0 & 0 \\ 0 & l \sigma_{\mathrm{m}}{ }^{2} & 0 & 0 \\ & & & \\ 0 & 0 & 1 \sigma_{\mathrm{p}}^{2} & 0 \\ 0 & 0 & 0 & 1 \sigma_{\theta}^{2}\end{array}\right]$

avec:

$-\sigma_{\mathrm{u}}{ }^{2}=\mathrm{h}^{2} \sigma_{\mathrm{y}}{ }^{2} ;-\sigma_{\mathrm{m}}{ }^{2}=v \sigma_{\mathrm{y}}{ }^{2} ;-\sigma_{\mathrm{p}}{ }^{2}=\left(\mathrm{r}-\mathrm{v}-\mathrm{h}^{2}\right)$

$\sigma_{y}^{2} ;-\sigma_{\theta}^{2}=(1-r) \sigma_{y}^{2}$;

- A : matrice de parenté;

- 1 : matrice identité;

$-\mathrm{h}^{2}$ : héritabilité;

- $r$ : répétabilité;

- $v$ : fraction de la variance phénotypique due à la composante "maternelle-élevage».

La matrice de parenté comprend l'ensemble des animaux, reproducteurs ou non, performeurs ou non. Les lignes de la matrice $Z$ correspondant aux animaux non performeurs ne contiennent que des 0 .

\section{Equations et résolution du modèle mixte}

Les équations du modèle mixte sont :

$\left[\begin{array}{cccc}X^{\prime} X & X^{\prime} Z & X^{\prime} W & X^{\prime} Z \\ Z^{\prime} X & Z^{\prime} Z+t_{1} A^{-1} & Z^{\prime} W & Z^{\prime} Z \\ W^{\prime} X & W^{\prime} Z & W^{\prime} W+t_{2} I & W^{\prime} Z \\ Z^{\prime} X & Z^{\prime} Z & Z^{\prime} W & Z^{\prime} Z+t_{3} \prime\end{array}\right]\left[\begin{array}{c}b \\ u \\ m \\ p\end{array}\right]\left[\begin{array}{l}X^{\prime} y \\ Z^{\prime} y \\ W^{\prime} y \\ Z^{\prime} y\end{array}\right]$

avec :

$-t_{1}=(1-r) / h^{2}$

$-t_{2}=(1-r) / v$;

$-t_{3}=(1-r) /\left(r-h^{2}-v\right)$.

Les effets $p$ et $m$ sont absorbés facilement grâce à la structure diagonale des matrices W'W $+t_{2} l$ et $Z^{\prime} Z+t_{3} l$. Le résultat de leur absorption se calcule directement à partir du nombre d'années de performances, des performances de chacun et de leurs sommes par individu et par mère. Les animaux non reproducteurs et les mères sont ensuite absorbés grâce à la structure particulière de l'inverse de la matrice de parenté. En regroupant les poulinières issues d'une même jument fondatrice (sans mère), on obtient une matrice "poulinière * poulinière" bloc diagonale dont l'inversion est aisée. De même, on regroupe les individus non reproducteurs par mère (Tavernier, 1988b). La matrice finale ne comporte plus que les effets fixes et les étalons. Elle est résolue par itération.

Les solutions des poulinières et des nonreproducteurs absorbés sont obtenues par "back-solving" des équations à l'aide des solutions étalons et effets fixes.

La précision de ces estimations est obtenue par approximation (Van Raden \& Freeman, 1985) de la décomposition par bloc de l'inverse de la matrice finale. Si on note l'inverse de la matrice finale :

$$
\left[\begin{array}{l}
H B^{\prime}-1 \\
B M
\end{array}\right]=\left[\begin{array}{l}
T S^{\prime} \\
S F
\end{array}\right]
$$

avec $\mathrm{H}$, bloc correspondant à la proche parenté de l'étalon dont on veut calculer la précision de l'estimation, on a :

$\mathrm{T}=\mathrm{H}^{-1}+\mathrm{H}^{-1} \mathrm{~B}^{\prime} \mathrm{F}$ B H $\mathrm{H}^{-1}$ 
Dans le sens ou $C \geq D$ veut dire que $(C-D)$ est positive semi-défnie, on peut montrer que :

- une borne supérieure de T est : $\mathrm{H}^{-1}+\mathrm{H}^{-1} \mathrm{~B}^{\prime}$ A B H ${ }^{-1}$;

- une borne inférieure de T est : $\mathrm{H}^{-1}$.

La borne supérieure de T est approchée en utilisant la structure connue de parenté du bloc $H$ et la parenté "moyenne" contenue dans $A$. Les précisions des estimations des poulinières et des non-reproducteurs sont obtenues simplement par décomposition par bloc de l'inverse et utilisation des précisions des étalons.

\section{Matériel}

Les gains en course des Trotteurs Français sont recueillis depuis de nombreuses années dans des annuaires publiés chaque année par la Société d'encouragement à l'élevage du cheval français. Ces résultats ne sont informatisés que depuis 1974. Les résultats antérieurs ont été saisis manuellement. Le fichier des performances est complet depuis les performances des naissances de 1966. La généalogie est apportée par le SIRE (Système d'Identification Répertoriant les Equidés). La généalogie des ancêtres de tous les performeurs a été remonté jusqu'aux environs de 1920-1940, y compris les Pur-Sang.

Le fichier final (performeurs nés après 1966 et leurs ancêtres) comprend 61012 chevaux, avec 42263 performeurs pour 120172 performances annuelles, 2828 étalons et 22250 poulinières. Parmi les reproducteurs nés après 1966, $99 \%$ des étalons (sur 676 ) et $56 \%$ des poulinières (sur 10 083) ont des résultats en course. Après le calcul sur ce fichier informatif, nous avons estimé le reste des chevaux de race Trotteur Français inscrits au SIRE, donc tous les non-performeurs et non-parents de performeurs nés depuis 1974 et leurs ancêtres, par la moyenne des estimations de leurs parents. Cela représente au total 140107 Trotteurs Français avec 2714 étalons Trotteurs Français et 35091 poulinières Trotteur Français.

Les paramètres génétiques avaient été estimés par Langlois (1985a) par analyse de variance sur le logarithme du gain par départ, divisé par la moyenne du gain par départ de la classe d'âge, de sexe, d'année du cheval. La répétabilité était de 0,38 de l'héritabilité de 0,23. Ces paramètres sont réestimés ici pour mettre en évidence l'effet maternel. Ils sont estimés par analyse de variance sur la performance précorrigée par une estimation des moindres carrés pour les effets fixes sur l'ensemble de notre fichier. L'héritabilité est de 0,26 , la répétabilité de 0,36 et la composante maternelle de 0,04 .

\section{RÉSULTATS}

Tous les résultats sont exprimés en points d'écart type phénotypique. Celui-ci est fixé à 20 points. La précision de l'indice est présentée sous forme de coefficient de détermination et varie de 0 à 1 .

\section{Estimation des effets fixes}

L'estimation des effets fixes âge-année obtenus par la résolution du modèle mixte qui fournit un BLUE (Best Linear Unbiased Estimate) est comparée à l'estimation des moindres carrés du même modèle ne comportant que les effets fixes (Fig. 1). La différence entre les 2 estimations est d'autant plus importante que l'année de performance augmente. L'écart est dû au progrès génétique qui est inclus pour les moindres carrés dans l'effet de l'année, alors qu'il doit s'en séparer comme c'est le cas pour le BLUE. De plus, à 6 ans, le BLUE de l'effet âge-année est toujours nettement inférieur à l'estimation par les moindres carrés : c'est que l'échantillon des chevaux qui courent à 6 ans est légèrement sélectionné. Leur avantage génétique est inclus dans les moindres carrés dans l'effet de l'âge, alors qu'il s'en détache dans le BLUE. Cette comparaison met en évidence l'influence des phénomènes, que nous allons mesurer par la 

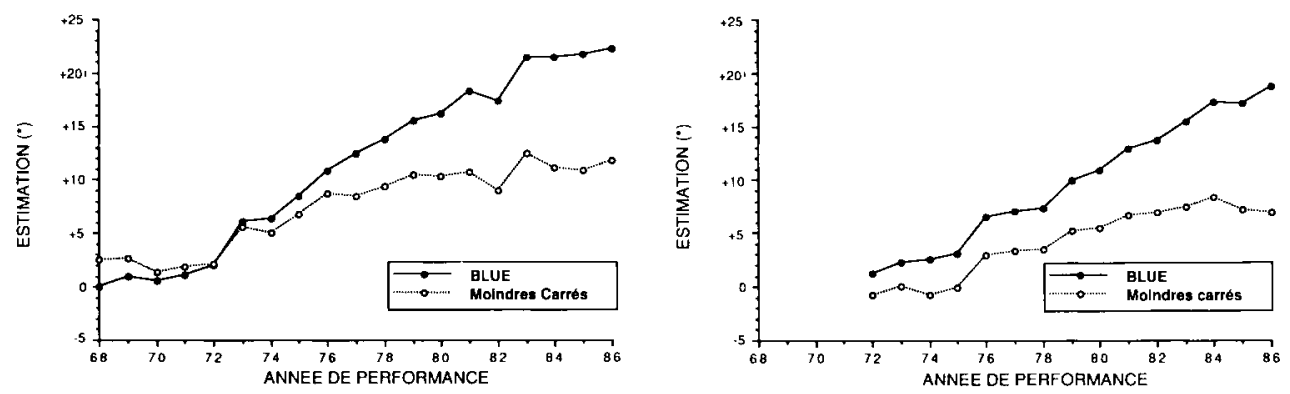

Fig. 1a et b. Estimation des effets fixes âge-années. a. Age 2 ans. b. Age 6 à 10 ans.

suite (progrès génétique, sélection des performeurs), sur l'estimation des effets fixes et justifie la nécessité de prendre en compte simultanément ceux-ci avec les effets génétiques.

\section{Comparaison avec les indices classi- ques}

Les indices BLUP ont été comparés aux indices classiques déjà calculés chez le Trotteur. Ces indices classiques sont des régressions de la valeur génétique de l'individu sur les performances de quelques apparentés choisis et précorrigés pour les effets fixes (Langlois, 1986). Pour les étalons, nous avons comparé l'indice BLUP à l'indice classique qui combine performances propres, performances des descendants et performances des conjointes. Cette corrélation est de 0,84 - valeur très hautement significative - (1 534 étalons concernés) avec des coefficients de détermination moyens de 0,72 pour l'indice BLUP et de 0,64 pour l'indice combiné. Elle est assez élevée, ce qui n'est pas surprenant pour des individus dont l'essentiel de l'information est contenu dans l'indice classique. Le BLUP n'apporte pas alors beaucoup d'informations supplémentaires. Il corrige cependant mieux pour le progrès génétique et pour les conjointes, qui n'intervenaient que par leurs propres perfor- mances dans l'indice classique. On observe donc quelques gros écarts pour des étalons de tête, avec une revalorisation des jeunes étalons.

Pour les chevaux uniquement performeurs, la comparaison a porté sur l'indice classique qui combinait seulement leurs propres performances. La corrélation est de 0,78 (valeur très hautement significative) (35 934 chevaux concernés) pour des coefficients de détermination moyens de 0,55 pour l'indice BLUP et de 0,42 pour l'indice de carrière. Cette corrélation est assez élevée et montre l'importance du test de performance dans la sélection du Trotteur Français. Avec des chevaux qui font en moyenne 2,8 années de gains et une répétabilité relativement faible par rapport à l'héritabilité, on a une bonne estimation du potentiel génétique d'après les performances.

Globalement, le BLUP apporte surtout un "plus" pour les chevaux sur lesquels on n'avait auparavant que peu de renseignements : poulinières et jeunes.

\section{Structure de la population}

Le " 0 " de référence correspond au niveau de la population de base, soit les chevaux de notre fichier dont on n'a pas remonté la généalogie des parents. 
Actuellement, la distribution des indices BLUP des naissances de 1986 (8 651 poulains) a pour moyenne $+11,4$ et pour écart type 6 . Le meilleur $a+35$ et le moins bon -9 . Un pour cent des chevaux sont au-dessus de $+26,10 \%$ au-dessus de +19 , le quart est supérieur ou égal à +15 . Les départements (Fig. 2) les meilleurs sont à plus d'un quart d'écart type audessus de la moyenne. Ce sont l'Orne (moyenne + 14 avec 1313 naissances) et le Calvados (moyenne + 13,4 avec 1215 naissances). Trois autres départements ont aussi des moyennes supérieures à l'ensemble de la France : la Mayenne ( $+12,8$ et 652 naissances), la Sarthe ( $+11,9$ et 383 naissances) et la Manche (+ 11,4 et 664 naissances). Parmi les étalons à la saillie en 1985 (pour produire les naissances de 1986), 16 étaient supérieurs à +30 et 137 à +20 . Leur seule utilisation suffisait pour créer la nouvelle génération (63 poulains par mâle).

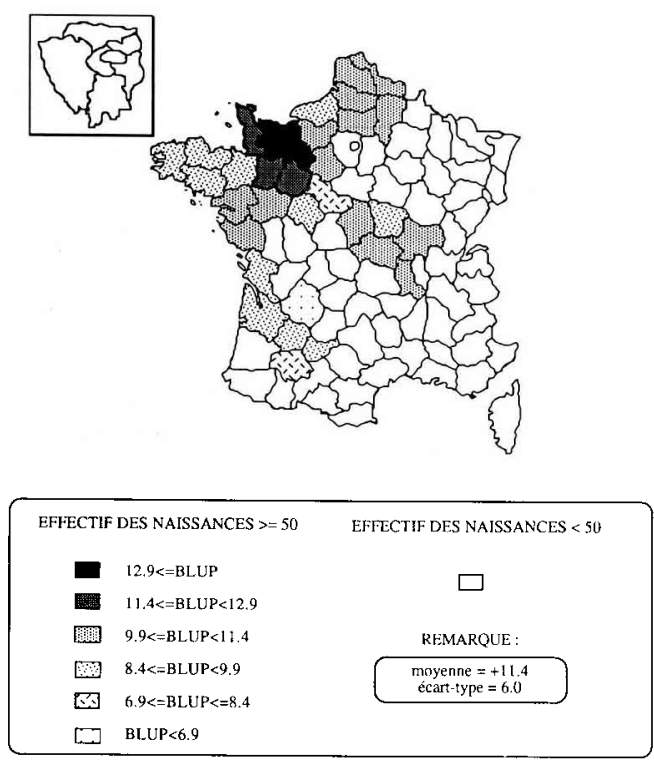

Fig. 2. Moyenne des indices BLUP des naissances des Trotteurs Français en 1986 par département.
Cependant, la distribution des étalons réellement utilisés a pour moyenne +13 ef pour écart type 7,9 , ils étaient 630 pour faire 8651 poulains (14 poulains par mâle). La distribution des poulinières a pour moyenne $+4,7$ et pour écart type 6,9. Une poulinière est dans les $10 \%$ meilleurs quand elle $a+14$, dans le quart meilleur quand elle $\mathbf{a}+9$.

\section{Progrès génétique}

Le progrès génétique est mesuré par l'évolution de l'indice BLUP en fonction de l'année de naissance. La Figure 3 représente ces évolutions de 1966 à 1986 dans l'ensemble de la population Trotteur Français du SIRE.

La supériorité des étalons par rapport à la population non reproductrice est de 14 points depuis le début des années 70 . Cette supériorité s'explique presque exclusivement par leur sélection sur performances propres. En effet, on peut mesurer la supériorité génétique obtenue par les performances de ces chevaux par rapport à leurs contemporains grâce à leur indice de carrière. L'indice de carrière est l'estimation de la supériorité génétique du cheval par régression à partir de ses per-

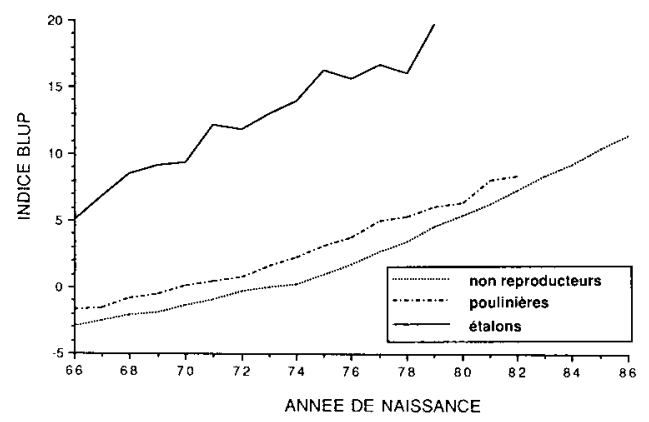

Fig. 3. Evolution génétique des Trotteur Français. 
formances propres. Pondéré par le nombre de descendants de chaque étalon, cet indice de carrière permet de mesurer l'influence génétique de l'étalon sur la population due à sa sélection sur performances. La moyenne pondérée des indices de carrière des étalons est de 13,5. La supériorité génétique totale donnée par le BLUP est donc constituée presque uniquement d'une supériorité sur performances.

Les poulinières ont une supériorité d'environ 1,5 point sur les non-reproducteurs. En revanche, elles ne sont pas sélectionnées elles-mêmes sur performances (Langlois, 1985b); elles le sont donc sans doute sur ascendance.

La supériorité de la génération suivante issue de ces reproducteurs par rapport à l'actuelle population est donc de $(14+1,5)$ / $2=7,8$ points. Avec un intervalle de génération de 11,6 ans (Langlois, 1985b), le progrès génétique annuel est de $7,8 / 11,6=$ 0,7 point. Ce progrès génétique espéré du fait de la supériorité des reproducteurs correspond bien au progrès génétique effectivement réalisé : le coefficient de régression de l'indice BLUP sur l'année de naissance est de 0,71 (valeur très hautement significative) de 1966 à 1986, soit $3,6 \%$ d'écart type phénotypique. Ce progrès est supérieur à celui réalisé chez les chevaux de CSO en France, qui est de $1,4 \%$ d'écart type phénotypique par an (Tavernier, 1988a). II demeure néanmoins inférieur à ce qui pourrait être réalisé sans modification profonde du système de production. Prenons comme exemple la populations des Trotteurs Français nés en 1983 , donc ayant pu être testés sur performances à 2 et 3 ans en 1985 et 1986 date limite des performances intervenant dans le calcul des BLUP - et en situation de sélection en 1986 (sans descendants). La moyenne des indices BLUP de cette génération est de 8,4. Si on ne gardait que les meilleurs $1 \%$ des mâles et $50 \%$ des femelles, ce qui est suffisant pour assurer le renouvellement de la population, la moyenne des indices BLUP des futurs reproducteurs pourrait être de 27,6 et 13,3 respectivement. Leur supériorité par rapport à la moyenne de leur génération est donc de 19,2 (contre 14 actuellement) et 4,9 points (contre 1,5 actuellement). Le progrès génétique à la génération suivante est de $(19,2+4,9) / 2=12$ points (contre 7,8 actuellement). De plus, ces reproducteurs sont sélectionnés plus rapidement qu'actuelle-ment : ils n'ont que 2 années de performances contre 4,6 pour les mâles et 2,8 pour les femelles aujourd'hui. L'intervalle de génération diminue et est égale à $[(11,6-2,6)+(11,6-0,8)] / 2=9,9$ ans (contre 11,6 actuellement). Le progrès génétique annuel est de $12 / 9,9=1,2$ point, soit $6 \%$ d'écart type phénotypique et $70 \%$ de progrès en plus par rapport à la situation présente.

\section{Estimation des non-gagnants}

Comparaison chevaux gagnants - chevaux non gagnants mais ayant couru chevaux n'ayant pas couru

On ne pouvait avoir une idée de la différence éventuelle de qualité entre les chevaux non gagnants sortis et non sortis en course ef le reste de la population à partir de la seule connaissance des performances basées sur les gains. L'indice BLUP permet maintenant de faire cette comparaison puisqu'il estime ces chevaux non gagnants à partir de leur parenté et qu'il les traite de la même manière, qu'ils aient ou on participé à des courses, ce qui évite les biais. 
Tableau I. Ecart génétique entre les chevaux gagnants, non gagnants et non sortis et leurs contemporains nés de 1974 à 1984.

\section{2 ans Performance réalisé à 3 ans et plus}

Ecart moyen en BLUP (\% effectif) des chevaux :

$\begin{array}{lrrrrrr}\text { Gagnants } & +2,0 & (7 \%) & +1,5 & (29 \%) & +3,7 & (15 \%) \\ \text { Non gagnants } & 0,0 & (5 \%) & 0,0 & (11 \%) & +2,1 & (4 \%) \\ \text { Non sortis } & -0,2 & (88 \%) & -0,8 & (60 \%) & -0,8 & (81 \%)\end{array}$

Un des problèmes du critère du gain est de ne pas attribuer de performances aux chevaux non titulaires de gains, qu'ils aient ou non participé à des courses. L'indice BLUP permet d'avoir une information à leur sujet à partir de leur parenté et de les comparer au reste de la population.

Le Tableau I représente la moyenne des valeurs génétiques des Trotteurs Français nés de 1974 à 1984 selon 3 catégories : les chevaux gagnants (= ayant des gains et pas forcément vainqueurs d'une épreuve), les chevaux non gagnants mais sortis, les chevaux non sortis et 3 classes d'âge de performances : 2, 3 et 6 ans et plus.

Dans tous les cas, la supériorité génétique réelle des chevaux sortis en course par rapport à l'ensemble des naissances est très inférieure à celle que sousentendent les effectifs. Ce ne sont pas les 12 ou 40 ou $19 \%$ meilleurs chevaux de leur génération à 2,3 et 6 ans qui sortent en course. On peut juste noter que :

- La qualification à 2 et à 3 ans n'est pas un signe de nette supériorité génétique : les chevaux qualifiés sans gains n'ont qu'un avantage de 1 point sur les chevaux qui ne sont pas du tout sortis. Cet avantage ne représente qu'un peu plus d'une année de naissance d'écart puisque le Trotteur Français progresse chaque année de 0,7 point. II y a plus de différence entre divers gagnants que globalement entre qualifiés et non-qualifiés.

- Ne pas sortir à 2 ans n'a pas de signification génétique.

- Ce n'est qu'à 6 ans qu'on ne garde en course que des chevaux un peu meilleurs que leurs contemporains.

\section{Estimation des poulinières sans perfor- mances}

Nous allons maintenant pouvoir comparer réellement les juments titulaires ou non de performances. Une distinction est faite entre jument qualifiée ou non dans les règlements de monte, ce qui ne semble pas correspondre à un réel écart de qualité au niveau de la production (Langlois, 1985b).

Pour étudier la jumenterie, nous avons analysé les mères des poulains nés en 1986 (Figs 4 et 5). Les poulinières qui ont eu des gains à un âge quelconque de leur vie représentent $50,5 \%$ de l'ensemble, celles qui sont sorties mais non gagnantes $21,3 \%$. II reste $28,2 \%$ de juments qui n'ont pas couru. Par rapport à leurs contemporaines nées la même année et devenues aussi poulinières, les juments gagnantes ont 2 points d'indice en plus de la moyenne, les juments non sorties ont 0,5 


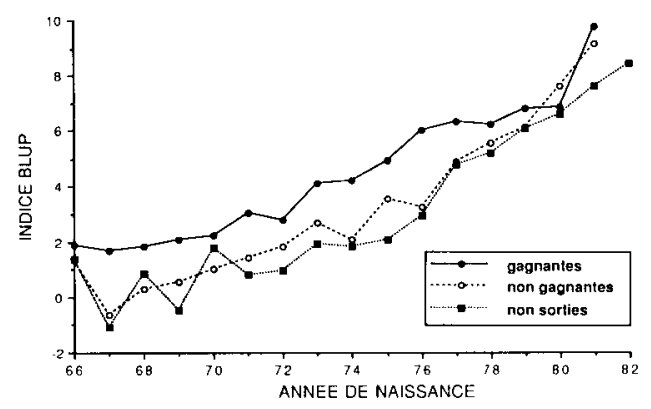

Fig. 4. Indice BLUP des juments Trotteur Français mères des naissances Trotteur Français de 1986.

point de retard et les juments sorties non gagnantes sont dans la moyenne. Mais les juments qui sortent en course arrivent beaucoup plus tard à la reproduction. Leur âge moyen est de 11,5 ans alors que celui des juments qui n'ont pas couru est de 9,4 ans et celui des juments qui ont couru mais n'ont pas gagné est, entre les deux, de 10,1 ans. Les 2 points d'indice en plus des juments gagnantes ne représentent qu'à peine 3 ans de progrès génétique, puisque l'on progresse chaque année de 0,7 point, soit 2,1 points en 3 ans. L'avantage des juments gagnantes par rapport à leurs contemporaines est donc grignoté par leur «retard" génétique par rapport aux juments plus jeunes qui sont à la reproduction en même temps qu'elles. C'est ce qui explique que la vraie moyenne des indices des juments gagnantes est de 4,7 , celle des non gagnantes de 4,5 et celle des juments non sorties de 4,5 également. Le test de sélection sur performance est donc actuellement peu efficace pour les juments trotteuses.

Cependant, il ne faut certainement pas abandonner l'idée de sélectionner les juments sur les performances; il faut juste revoir son application. Tout d'abord, il convient d'effectuer un retour rapide à l'éle-

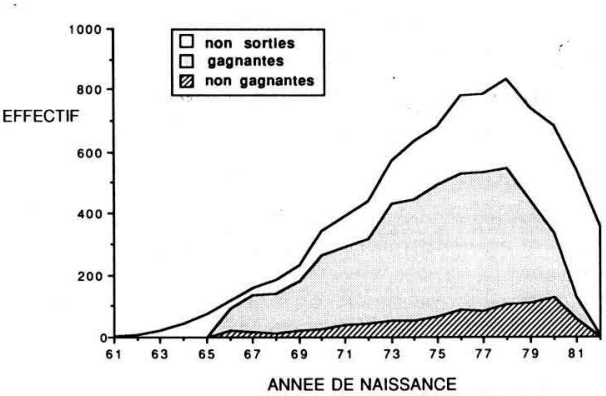

Fig. 5. Année de naissance des juments Trotteur Français mères des naissances Trotteur Français de 1986.

vage pour ne pas se laisser rattraper par la génération montante. Deux solutions sont possibles. La première est l'utilisation de la transplantation embryonnaire qui permet à une jument d'avoir des descendants tout en continuant sa carrière sportive. La seconde est de n'utiliser que des carrières en course courtes. En effet, les corrélations génétiques entre performances réalisées à des âges différents sont élevées : 0,80 entre 2 ans et 3-4 ans, 0,90 entre 3 ans et plus (Langlois, 1984). Cela permet d'obtenir un progrès génétique pour le caractère «aptitude au trot à l'âge mûr" important tout en sélectionnant à des âges précoces. Une petite réserve est à apporter aux performances réalisées à 2 ans, qui sont moins bien corrélées avec les performances réalisées à 5 et 6 ans $(0,45)$. Ensuite, il faut effectuer une réelle sélection sur la performance : seules les meilleures juments de course doivent revenir à l'élevage. Si on ne conservait réellement que les $50 \%$ meilleures juments sorties en course, elles auraient avec une seule année de performance une supériorité de 4 points par rapport aux juments qui n'auraient pas couru, et même en arrivant à l'élevage avec un an de retard par rapport à celles-ci, elles conserveraient nettement leur supériorité. 
L'indice BLUP doit donc devenir un véritable outil de gestion de la jumenterie. II permet la comparaison permanente de la qualité de reproducteur des juments à l'élevage avec celle des nouvelles venues qui bénéficient, dans une population qui évolue rapidement, du progrès génétique.

\section{Homogamie}

II est préférable, pour optimiser ses chances de produire un bon cheval, d'accoupler un bon étalon à une bonne jument. L'importance de ce phénomène peut se mesurer grâce à la corrélation entre l'indice BLUP des 2 conjoints. Cette corrélation est de 0,44 sur l'ensemble de notre fichier qui recense 136753 couples. L'homogamie est donc bien présente dans la population des Trotteurs Français et justifie d'autant plus la prise en compte du niveau du harem pour estimer un étalon, et réciproquement des conjoints pour estimer une poulinière. En revanche, elle est difficile à interpréter. Elle est bien superieure à la corrélation phénotypique qui est de 0,15 . Elle est sans doute le résultat d'une prise de décision complexe qui inclut performances, origines et localisation géographique.

\section{CONCLUSION}

L'intérêt de l'indice BLUP en modèle animal dans les populations chevalines est, ici encore, vérifié : indexation généralisée, estimation plus précoce, prise en compte de toutes les performances de toute la parenté, estimation des chevaux restés à l'élevage. La structure actuelle de la population donne l'image d'une production très régionale, axée sur la Basse-Normandie et le Maine. II y a une recherche en matière de sélection (progrès génétique de 3,5\% d'écart type phénotypique) et de gestion des accouplements (corrélation de 0,44), mais encore limitée au regard des possibilités démographiques. Le Trotteur peut avoir un avenir plus brillant encore que son passé. Pour cela, il doit se débarrasser des préjugés attachés à la valeur de la jumenterie non performeuse (supposée inférieure), aux performances précoces (supposées faiblement corrélées aux autres) et à l'intérêt de la multiplicité des étalons (supposée bénéfique ?). Le BLUP est la meilleure estimation actuelle du potentiel génétique des Trotteurs Français, il est le critère de sélection sur lequel on doit se baser pour créer une structure de sélection efficace. Cette efficacité sera démultipliée avec l'utilisation de l'insémination artificielle ou le transfert d'embryon qui peuvent permettre d'une part, une rotation plus rapide des reproducteurs, avec la mise à la reproduction d'individus qui peuvent continuer une carrière sportive, et d'autre part, la diffusion de la supériorité génétique avec l'augmentation du nombre de descendants par reproducteurs confirmés. Un progrès de plus de $50 \%$ est facilement envisageable.

\section{RÉFÉRENCES}

Arnason T., Bendroth M., Phillipson J., Henrikson K. \& Darenius A. (1988) Genetic evaluation of Swedish Trotters. Proc. VI World Conference on Animal Production, Helsinki, Finland, June 27-July 1, 1988, EAAP Special Symposium, Estimation of breeding value in Trotters

Katona O. \& Distl O. (1988) SIRE evaluation in the German Trotter (Standardbred) population. Proc. VI World Conference on Animal Produc- 
tion, Helsinki, Finland, June 27-July 1, 1988, EAAP Special Symposium, Estimation of breeding value in Trotters

Klemetsdal G. (1988) Norvegian Trotter breeding and estimation of breeding value. VI World Conference on Animal Production, Helsinki, Finland, June 27-July 1, 1988, EAAP Special Symposium, Estimation of breeding value in Trotters

Langlois B. (1984) Héritabilité et corrélations génétiques des temps records et des gains établies par les Trotteurs Français de 2 à 6 ans. Journées d'étude et de communication chevalines de la Fédération européenne de zootechnologie. La Haye, 6-9 août

Langlois B. (1985a) Etude d'un exemple d'application de la méthode d'indexation pour les chevaux de course : le cas du Trotteur Français. Polycop. Stn. Génét. Quant. et Appl., CNRZ

Langlois B. (1985b) Effets de la sélection sur les données de performances chez le Trotteur Français. In: Proc. Journée de la Recherche Chevaline. CEREOPA, Paris, 6 mars 1985

Langlois B. (1986) Evaluation génétique des Trotteurs Français d'après leurs gains. In: Proc. Journée de la Recherche Chevaline. CEREOPA, Paris, 12 mars 1986

Langlois B. (1988) Breeding evaluation of French Trotters according to their race earnings. 1. State of the situation. Proc. VI World Conference on Animal Production, Helsinki, Finland, June 27-July 1, 1988, EAAP Special Symposium, Estimation of breeding value in Trotters

Leroy P. (1988) Estimation of Breeding values of Belgian Trotters using an animal model. Proc. VI World Conference on Animal Production, Helsinki, Finland, June 27-July 1, 1988, EAAP Spe- cial Symposium, Estimation of breeding value in Trotters

Minkema D. (1988) Breeding value estimation of Trotters in the Netherlands. Proc. VI World Conference on Animal Production, Helsinki, Finland, June 27-July 1, 1988, EAAP Special Symposium, Estimation of breeding value in Trotters

Ojala M. (1988) Breeding evaluation of Trotters in Finland. Proc. VI World Conference on Animal Production, Helsinki, Finland June 27-July 1, 1988, EAAP Special Symposium, Estimation of breeding value in Trotters

Tavernier A. (1988a) Application du “modèle animalı dans l'évaluation génétique des chevaux de sport. In : Proc. Colloque International Amélioration Génétique du Cheval de Sport. CEREOPA, Paris, 9 mars 1988

Tavernier A. (1988b) Advantages of BLUP animal model for breeding value estimation in horses. Livest. Prod. Sci. 20, 149-160

Tavernier A. (1988c) Breeding evaluation of French Trotters according to their race earnings. 2. Prospects. Proc. VI World Conference on Animal Production, Helsinki, Finland, June 27-July 1, 1988, EAAP Special Symposium, Estimation of breeding value in Trotters

Tolley E.A., Notter D.R. \& Marlowe T.J. (1988) The environmental effects of pace of race and purse for 2 and 3-year old standardbred Trotters. Proc. VI World Conference on Animal Production, Helsinki, Finland, June 27-July 1, 1988, EAAP Special Symposium, Estimation of breeding value in Trotters

Van Raden P.M. \& Freeman A.E. (1985) Rapid method to obtain bounds on accuracies and prediction error variances in mixed models. J. Dairy Sci. $68,2113-2133$ 